\title{
Tyrosinkinaseinhibition beim metastasierten RCC
}

\author{
Retrospektiv wurde die Effizienz von \\ Sunitinib und Pazopanib in der Erst- \\ linien- und Zweitlinientherapie des \\ metastasierten Nierenzellkarzinoms \\ (mRCC) verglichen.
}

\begin{abstract}
n der Erstlinientherapie des mRCC sind Sunitinib (SUN) und Pazopanib (PAZ) mittlerweile Standard. SUN verbessert im Vergleich zu Interferon a das progressionsfreie Überleben (PFS) und tendenziell auch das Gesamtüberleben (OS). PAZ verlängert gegenüber Placebo das PFS und tendenziell auch das OS. In der retrospektiven Datenbankanalyse wurden 7.438 Patienten mit mRCC betrachtet, die in der Erstlinie SUN $(\mathrm{n}=6.519)$ oder PAZ $(\mathrm{n}=919)$ erhalten hatten. Das mediane Follow-up betrug 40,4 Monate. Hinsichtlich der prognostischen Gruppen (nach International Metastatic Renal-Cell Carcinoma Database Consortium [IMDC]) waren die Therapiegruppen ausgeglichen ( $\mathrm{p}=0,36)$ : günstige Pro-
\end{abstract}

gnose $23 \%$ bei SUN und $24 \%$ bei PAZ, intermediär $57 \%$ und $58 \%$ sowie ungünstig $20 \%$ und $18 \%$. Mehr mit SUN behandelte Patienten begannen mit der Therapie innerhalb eines Jahres nach Diagnose.

Die Gruppen unterschieden sich hinsichtlich des medianen OS nicht: 22,3 Monate unter SUN versus 22,6 Monate unter PAZ $(\mathrm{p}=0,65)$. Nach Adjustierung für die IMDC-Kriterien betrug die $\mathrm{Ha}$ zard Ratio (HR) für Tod bei PAZ versus SUN 1,03 ( $\mathrm{p}=0,58)$. Auch beim PFS gab es keinen Unterschied (SUN vs. PAZ: 8,4 vs. 8,3 Monate; $\mathrm{p}=0,17 ; \mathrm{HR} 1,08$; $\mathrm{p}=0,12)$. Die Ansprechraten waren ebenfalls vergleichbar (SUN vs. PAZ: 30 vs. $28 \%$; $\mathrm{p}=0,15$ ).

Von den first-line mit SUN behandelten Patienten erhielten mehr eine Zweitlinientherapie ( $49 \%)$ als von den zuerst mit PAZ Behandelten (38\%; $\mathrm{p}<0,0001)$. Allerdings erhielten mehr Patienten zum Zeitpunkt der Analyse PAZ noch immer als Erstlinientherapie. Klammerte man die Patienten aus, die noch immer firstline behandelt wurden, erhielten 51,5\% der SUN- bzw. 51,2\% der PAZ-Gruppe eine Zweitlinientherapie. Es ergab sich kein Unterschied in der Zweitlinie zwischen der Post-SUN- und der Post-PAZGruppe hinsichtlich OS (13,1 vs. 11 Monate; $\mathrm{p}=0,27)$ oder PFS (3,7 vs. 5,0 Monate; $\mathrm{p}=0,07)$.

Fazit: Im bevölkerungsbasierten Setting zeigten SUN und PAZ bei mRCC-Patienten vergleichbare Effizienz und beeinflussten die Prognose einer nachfolgenden Zweitlinientherapie nicht. Jüngste Daten zur individuellen Dosisanpassung, um die Toxizität der Therapie zu reduzieren, wurden in dieser Studie nicht beachtet. Dieser Aspekt sollte in weiteren Studien untersucht werden.

Kathrin von Kieseritzky

Ruiz-Morales MJ et al. First-line sunitinib versus pazopanib in metastatic renal cell carcinoma: Results from the International Metastatic Renal Cell Carcinoma Database Consortium. Eur J Cancer. 2016; 65: 102-8.

\section{Blasenkarzinom: progredient oder nicht?}

\section{Ist es möglich, anhand eines Tumor-Genexpressionsprofils von progredienten und nicht progredienten T1G3-Blasenkarzinomen eine Signatur zur Vorhersage des Progressionsrisikos zu identifizieren? Dieser Frage wurde in einer Studie nachgegangen.}

Z wei Drittel der Urothelkarzinome, der häufigsten Form von Blasenkrebs, sind bei Diagnose noch nicht in die Muskulatur vorgedrungen. Jedoch zeichnet sich das nicht muskelinvasive Blasenkarzinom durch ein hohes Rezidivrisiko aus: In Abhängigkeit vom Tumorstadium und -grad rezidivieren nach transurethraler Resektion (TUR) 30-85\% der Tumoren, bis zu $17 \%$ wachsen muskelinvasiv. Die Progression erfolgt meist innerhalb von 48 Monaten nach Erstdiagnose und ist abhängig vom Tumorstadium, Tumorgrad und der Anwesenheit begleitender Carcinoma in situ (CIS).

In dieser retrospektiven Studie wurde die Genexpression in Tumorproben von 96 Patienten mit Blasenkarzinom im
Stadium T1G3 ohne CIS ermittelt, die sich einer TUR unterzogen hatten. Fast alle Patienten hatten zudem einen Induktionszyklus BCG (Bacillus-Calmette-Guérin) erhalten. 56 Patienten entwickelten nicht muskelinvasive Rezidive, bei 40 erfolgte eine Progression zu einem muskelinvasiven Blasenkarzinom. Die mediane Zeit bis zur Progression betrug ein Jahr, das mediane Follow-up bei den Patienten mit nicht muskelinvasivem Tumor 4,8 Jahre. Ein Tumordurchmesser von $\geq 3 \mathrm{~cm}$ fand sich bei 9 der 56 Patienten (16\%) mit nicht muskelinvasivem und 6 der 40 Patienten (15\%) mit muskelinvasivem Rezidiv.

Insgesamt wurden 1.294 Gene ermittelt, die bei progredienten und nicht progredienten Tumoren unterschiedlich stark exprimiert waren. Es kristallisierte sich eine Expressionssignatur von fünf Genen heraus, anhand derer Patienten mit progredientem von solchen mit nicht progredientem Tumor unterschieden werden konnten: ANXA10, DAB2, HYAL2, SPOCD1 und MAP4K1.

Die Sensitivität des Tests lag bei 79\%, die Spezifität bei $86 \%$ (positiver prädiktiver Wert: $90 \%$; negativer prädiktiver Wert: $71 \%$; Fehlerrate: 19\%; AUC [,,area under the curve"] 0,83). Eine direkte Interaktion zwischen den fünf Genen des Modells konnte nicht festgestellt werden.

Fazit: Progrediente und nicht progrediente T1G3-Harnblasenkarzinome zeigen unterschiedliche Genexpressionsmuster. Die in der Studie herausgearbeitete Expressionssignatur von fünf $\mathrm{Ge}$ nen eignet sich offenbar dafür, Patienten mit einem hohen Risiko für Progression zu identifizieren. Kathrin von Kieseritzky

Van der Heijden AG et al. A five-gene expression signature to predict progression in T1G3 bladder cancer. Eur J Cancer. 2016; 64: 127-36. 